\title{
CARACTERIZACIÓN PROSÓDICA DEL ESTILO DIRECTO DE HABLA EN LA CONVERSACIÓN COLOQUIAL
}

\author{
ADRIÁN CABEDO NEBOT \\ Universitat de València. Grupo Val.Es.Co. \\ Adrian.Cabedo@uv.es
}

\section{Resumen}

Este artículo pretende caracterizar desde un punto de vista prosódico el estilo directo de habla que podemos encontrar de modo habitual en cualquier intervención de una conversación coloquial. Así pues, nuestro propósito es comparar fragmentos de estilo directo con fragmentos normales de habla ( $\sin$ estilo directo), para poder extraer conclusiones de carácter prosódico. En ese sentido, hemos analizado la frecuencia fundamental media, el rango tonal, la duración y la velocidad de habla de grupos entonativos procedentes de una conversación coloquial.

PALABRAS CLAVE: estilo directo, conversación, prosodia, frecuencia fundamental,
\end{abstract} curva melódica.

\begin{abstract}
This paper tries to characterize direct speech in any colloquial conversation from a prosodic point of view. Thus, its aim is to compare direct speech parts with other speech parts, in order to extract prosodic conclusions. Regarding this, we have analyzed pitch, tonal rank, length of sound and speech velocity from some colloquial conversation tonic groups.

KEYWORDS: direct speech, conversation, prosody, pitch, tonic group.
\end{abstract}

\section{Introducción ${ }^{1}$}

Este artículo pretende aproximarse a la caracterización prosódica del estilo directo de habla en la conversación coloquial. En este sentido, diremos que una conversación es coloquial cuando presente los siguientes rasgos (Briz y Grupo Val.Es.Co., 2002):

a) Carácter no planificado de los enunciados.

b) Finalidad no transaccional, es decir, se prioriza el carácter fático de la interacción.

c) Tono informal.

d) Relación de igualdad social y funcional.

e) Relación vivencial de proximidad.

f) Marco de interacción familiar.

g) Temática no especializada.

1 Este artículo ha contado con la subvención del proyecto GV/2007/160, titulado "Transcripción y digitalización del Corpus Val.Es.Co", de la Generalitat Valenciana. 
En toda conversación coloquial, asimismo, es posible encontrar diferentes tipos de enunciados. En ocasiones, pueden aparecer construcciones inacabadas ( $y$ entonces me dijo...), reformulaciones (Lo que digo- quiero decir que...), llamadas al interlocutor (Ye, Mira...), etc. De todas ellas, una de las construcciones oracionales más características es el estilo directo de habla (en adelante, ED), que acontece cuando se: "reproducen textualmente las palabras o los pensamientos de los personajes sin intervenir (...) los verbos introductores suelen ser (...) declarativos, de percepción o similares (...) decir, preguntar, contestar..." (Antas, 2005: 115). Un ejemplo sería:

(1) y le dijo dice bueno/ esto puede pasar// dice porque $\rightarrow$ si fuese mayor $\uparrow$ aún aún (Briz y Grupo Val.Es.Co., 2002: 192).

Mediante el ED, el hablante consigue, de algún modo, dramatizar sus elocuciones, al vincular el dictum propio con el dictum de otros, es decir, al interpretar las palabras de otros interlocutores en un momento determinado (pasado, correlativo o futuro ${ }^{2}$ ). Por tanto, se trata de un procedimiento muy utilizado en la conversación coloquial, dado que actúa como soporte dramático de nuestros actos locutivos.

\section{Unidades de estudio}

Los mecanismos de segmentación que se utilizan en la conversación coloquial vienen, a menudo, determinados por diferentes índices: marcadores del discurso, ascensos o descensos tonales, etc. (Hidalgo, 2006). En la teoría de unidades del grupo de investigación Val.Es.Co. (Valencia, Español Coloquial), se señalan diferentes tipos de unidades vinculadas a la segmentación del discurso hablado en el nivel monológico; entre ellas, el acto y el subacto. El acto se caracteriza por: “(a) valor modal completo, una única función ilocutiva específica (pregunta, rechazo, etc.); y (b) unidad melódica, ya que su curva entonativa resulta completa en sí misma (la aseveración por ejemplo presenta un tonema descendente de carácter final)" (Hidalgo, 2006: 138). Por su parte, el subacto ${ }^{3}$ es una "unidad monológica estructural, constituyente inmediato del acto, caracterizada por constituir un segmento informativo e identificable en una conversación" (Briz y Grupo Val.Es.Co., 2002: 47).

Los fragmentos de ED constituyen, por sí mismos, actos de habla específicos en el desarrollo de toda intervención. No obstante, estos actos de habla pueden presentar dos circunstancias: actos compuestos por un único segmento de habla e informativo (actos simples) o actos compuestos por dos o más segmentos de habla (actos complejos, formados por más de dos subactos). Veamos algunos ejemplos:

\footnotetext{
2 Un ED será futuro siempre que pretenda anticiparse a lo que otro hablante diría en una situación contextual determinada. Por ejemplo: ¡Qué coño es esto! dirá si le regalamos este peluche para su cumpleaños.

${ }^{3}$ Los subactos, a su vez, pueden clasificarse en: sustantivos directores (poseen contenido proposicional y portan la fuerza ilocutiva de la intervención), sustantivos subordinados (contenido proposicional dependiente de un sustantivo director) y adyacentes (compuestos por elementos que aportan información extraproposicional, por ejemplo: modalizadores de tipo entonces, ¿sabes?...) (Briz y Grupo Val.Es.Co., 2002: 50).
} 
(2) C: ¿y a quién ha pagao?

(3) J: pero ¿qué vas a hacer $\uparrow$ ? ¿algún arte marcial o pesas?

En (2) tenemos un único acto (fuerza ilocutiva interrogativa, curva melódica completa), mientras que en (3) tenemos dos subactos: uno sustantivo director (pero ¿qué vas a hacer $\uparrow$ ?) y otro sustantivo subordinado (¿algún arte marcial o pesas?).

Por otro lado, algunas propuestas tradicionales (Navarro Tomás, 1944; Wennestrom 2001) y otras más actuales (Hidalgo y Padilla, 2006; Hidalgo, 2006) vinculan la presencia de tonemas ascendentes con un mecanismo de encajonamiento semántico en el discurso hablado. Así pues, la presencia del tonema ascendente $(\uparrow)$ en el ejemplo (3) indica que todavía falta un segundo segmento de habla que complete la semanticidad del acto completo. Desde esta perspectiva, el comportamiento tonal es indicativo de la posible división secuencial de los enunciados entre sí y de sus partes.

En realidad, otro fenómeno prosódico que se ha postulado últimamente como elemento auxiliar para la segmentación de los enunciados es el reajuste tonal (Garrido, 2002). Según el reajuste tonal, el principio tonal de cada grupo entonativo ${ }^{4}$ puede ser total (F0 inicial mayor que la F0 final del grupo entonativo anterior) o parcial (F0 inicial menor o igual que la F0 final del grupo entonativo anterior). Si mantenemos una posible correlación entre lo prosódico y lo semánticopragmático, podríamos sugerir que el reajuste total marca el paso entre actos y que, por su parte, el reajuste parcial hace lo propio con los subactos internos de un acto complejo. Más adelante, veremos que no siempre es así.

\section{Objetivos}

Una vez señaladas las unidades de estudio (acto, subacto, grupo entonativo) que vamos a utilizar para la presente investigación, conviene delimitar los objetivos que pretendemos alcanzar:

a) Identificar la diferencia entre fragmentos de ED y fragmentos de habla habituales. En adelante, nos referiremos a las secuencias orales que no estén compuestas por estilo directo como fragmentos habituales o normales de habla; no tanto porque consideremos que estas son más habituales que las otras con ED, sino porque nos sirve como mecanismo diferenciador en este estudio.

b) Analizar el paso de secuencias de habla normales a secuencias de ED. Esto se conseguirá analizando, por un lado, el reajuste tonal y, por otro, la delimitación anterior de los grupos entonativos (introducidos por inflexión tonal o pausa).

c) Señalar la correlación entre tipos de reajuste tonal y segmentos semánticopragmáticos introducidos (actos o subactos).

\footnotetext{
4 Entendemos por grupo entonativo: "porción de discurso comprendida entre dos pausas e inflexión del fundamental, entre inflexión del fundamental y pausa, o entre dos inflexiones del fundamental que configura una unidad sintáctica más o menos larga o compleja (sintagma, cláusula, oración)" (Quilis et alii, 1993: 56-57).
} 


\section{Metodología}

En principio, los fragmentos analizados en esta investigación han sido extraídos del corpus publicado por Briz y Grupo Val.Es.Co. en 2002. En concreto, se han analizado 5 intervenciones con fragmentos de ED en su interior. A su vez, estas intervenciones pertenecen únicamente a una hablante.

Tomemos, como ejemplo, dos de las intervenciones que hemos utilizado en este estudio:

(4) C: ah sí// pues esõ mee- una compañera me dice ;hala!/ Carmen/ ¿te vienes a- a esto de los libros que nos regalan una cosita de sumar $\rightarrow /$ o de no sé qué HISTORIA? (Briz y Grupo Val.Es.Co.,2002: 191).

(5) P: y- y le dijeron// lo llevó Mari Ángeles a un ciiirujano $\rightarrow$ y le dijo dice bueno/ esto puede pasar// dicẹporque $\rightarrow$ si fuese mayor $\uparrow$ aún aún/ pero aún es pequeñito// pero luego lo he llevao a éste y dice NOO/ si fuese de ombligo $\uparrow /$ le dejaríamos que el niño $\rightarrow$ (Briz y Grupo Val.Es.Co., 2002: 192).

En otro sentido, podríamos haber optado por utilizar intervenciones de más hablantes o, por el contrario, más intervenciones de la hablante seleccionada. Aun así, consideramos que esta muestra poblacional ${ }^{5}$ es representativa y que responde a la finalidad de esta investigación, es decir, a una caracterización aproximativa del estilo directo de habla en la conversación coloquial.

Asimismo, estas intervenciones han sido secuenciadas en grupos entonativos (en adelante, GE), por lo que, finalmente, se han analizado un total de 50 GE para alcanzar los resultados que se presentan en el siguiente apartado.

De ellos, se han tomado las siguientes variables:

a) Grupo entonativo introducido por reajuste total o parcial. Se han excluido, por tanto, los grupos entonativos iniciales de intervención, dado que, por posición, no presentan un reajuste frecuencial con ningún GE anterior.

b) F0 inicial, final, máxima, mínima y media de cada grupo entonativo. Con este análisis se ha querido investigar si existe o no diferencia tonal entre fragmentos con ED y fragmentos habituales.

c) Diferencia frecuencial con el GE anterior. Esta puede ser negativa (-) si el reajuste es parcial o positiva (+) sí el reajuste es total.

d) Rango tonal. Nos referimos con esta referencia terminológica a la distancia entre la F0 mínima del GE y la F0 máxima, es decir, al espacio tonal por el que transcurre la curva melódica y que, al fin y al cabo, determina la amplitud tonal de los fragmentos de habla, sean con ED o sin ED.

\footnotetext{
${ }^{5}$ En terminología estadística, entendemos por población: "el universo de objetos al cual se refiere el estudio que se pretende realizar (...). Por ejemplo, el conjunto de hogares o unidades de gasto (...), el grupo de alumnos y alumnas de una clase, los automóviles fabricados en una factoría durante un mes..." (Peña y Romo, 1999: 8). Así pues, en nuestro estudio, la población se refiere al número de grupos entonativos analizados.
} 
e) Delimitación anterior. Si analizamos cómo se delimitan los grupos anteriores y posteriores podemos llegar a diferentes conclusiones: en primer lugar, podemos saber si es frecuente que haya una pausa anterior al fragmento con ED del tipo Juan dijof Mecagüen mis muertos o, por el contrario, únicamente una inflexión del fundamental marca el paso de un grupo a otro. Ej: Juan dijo Mecagüen mis muertos.

f) ED. Con esta variable pretendemos averiguar cuántos grupos pertenecen al ED y cuántos no. Como veremos, hay un reparto equitativo entre ambos.

Finalmente, el estudio estadístico de las variables, tanto a nivel individual como vinculado, nos permitirá establecer ciertas apreciaciones sobre el carácter prosódico del ED en la conversación coloquial. Así pues, el estudio estadístico se ha realizado con el uso del software SPSS 15, que nos ha proporcionado información de interés acerca de nuestro objeto de estudio.

El estudio vinculado de las variables utilizadas en esta investigación deberá responder a las siguientes preguntas:

a) ¿Existe alguna diferencia tonal entre fragmentos habituales y fragmentos con ED?

b) ¿Cómo se introducen las secuencias de ED en el discurso?

c) ¿Existe alguna relación entre el tipo de reajuste y el tipo de unidad (acto o subacto) introducida?

En el siguiente apartado, intentaremos desvelar algunas de estas incógnitas.

\section{Resultados}

\subsection{Media y rango tonal}

Si se pretende establecer la singularidad de ED en la conversación coloquial hay que perseguir una comparación con fragmentos habituales de habla, de manera que las peculiaridades de las secuencias con ED tengan marcas identificativas que las diferencien o, como mínimo, las particularicen respecto a otras secuencias de habla.

Por tanto, el primer paso ha sido investigar la configuración tonal de las intervenciones del corpus para poder, de algún modo, caracterizar los grupos entonativos con y sin ED. En este sentido, hemos analizado la F0 media y el rango tonal de los GE de nuestras intervenciones para encontrar las pecualiaridades que singularizan los fragmentos con ED de otros. Los resultados han sido los siguientes:

\footnotetext{
${ }^{6}$ En el sistema de transcripción del grupo Val.Es.Co las pausas cortas inferiores al medio segundo se marcan con un $\langle/\rangle$, las que se encuentran entre medio segundo y un segundo con $\langle/|>$, si ocupan más de un segundo, con $</ / />$. (Briz y Grupo Val.Es.Co., 2002).
} 


\begin{tabular}{|c|c|c|c|c|}
\hline & & Media $^{7}$ & Mediana & $\begin{array}{l}\text { Diferencia } \\
\text { porcentual }\end{array}$ \\
\hline \multirow{2}{*}{$\begin{array}{c}\text { F0 } \\
\text { media }\end{array}$} & No ED & $184,34 \mathrm{~Hz}$ & $193,12 \mathrm{~Hz}$ & \multirow{2}{*}{$\begin{array}{l}21,71 \% \text { (media) } \\
19,17 \text { (mediana) }\end{array}$} \\
\hline & Si ED & $235,37 \mathrm{~Hz}$ & $230,15 \mathrm{~Hz}$ & \\
\hline \multirow[b]{2}{*}{$\begin{array}{c}\text { Rango } \\
\text { tonal }\end{array}$} & No ED & $76,03 \mathrm{~Hz}$ & $66,32 \mathrm{~Hz}$ & \multirow{2}{*}{$\begin{array}{l}75,41 \% \text { (media) } \\
96,27 \% \\
\text { (mediana) }\end{array}$} \\
\hline & Si ED & $133,37 \mathrm{~Hz}$ & $130,17 \mathrm{~Hz}$ & \\
\hline
\end{tabular}

Figura 1. F0 media de GE con y sin ED

De entrada, pueden apreciarse sustanciales diferencias. En primer lugar, la F0 media de los GE que no forman parte de estilo directo presentan registros tonales menores que los que sí forman parte de ED; en concreto, el incremento de media es del 21,71\%. Este dato es importante, ya que, de alguna manera, viene a sostener la opinión tradicional de que existe una cierta ponderación tonal $\mathrm{o}$, incluso, una modelización de la voz en fragmentos que introducen estilo directo.

Como puede observarse, el rango tonal es claramente diferente. En GE que pertenecen a secuencias de ED, se aprecia prácticamente un aumento en el espacio tonal de casi el 100\%, es decir, que la amplitud tonal de los grupos entonativos del estilo directo es claramente superior a los de tipo habitual. Así pues, esta circunstancia contribuye a mantener lo dicho anteriormente, ya que, en cierto sentido, el ED introduce un marcado carácter dramático y ponderativo de las elocuciones.

\subsection{Delimitación anterior, velocidad de habla y posición frecuente}

Como se ha comentado con anterioridad, el reajuste tonal supone que el inicio de un GE tiene un valor tonal en $\mathrm{Hz}$ superior o inferior al último valor tonal del GE anterior ${ }^{9}$. Esto nos lleva a preguntarnos si existe alguna relación entre el tipo de reajuste que aparezca (total o parcial) y la delimitación pragmasintáctica (en actos y subactos) del discurso.

En principio, según la concepción de grupo entonativo que utilizamos en este estudio, la delimitación prosódica de estos puede realizarse mediante una inflexión tonal o pausa

\footnotetext{
${ }^{7}$ La media es el "promedio que formaliza el concepto intuitivo de punto de equilibrio o centro de gravedad de las observaciones" (Pena y Romo, 1999: 50), mientras que la mediana es "una medida de posición que expresa el centro de los datos como el punto que separa las observaciones de menor a mayor" (Pena y Romo, 1999: 66). En general, la media es un número relativo obtenido a partir de la suma de todos los valores de un grupo dividido por el número total de valores, mientras que la mediana es el número de un único valor que se articula como eje central de la población, es decir, que por encima suyo está la mitad numérica superior y, por debajo, la mitad numérica inferior. Por ejemplo, 3 es la media de sumar $(2+1+6)$ y luego dividirlo por 3 ; mientras que 5 es la mediana del grupo $(1,2,3,4,5,6,7,8,9)$.

${ }^{8}$ Estas mediciones se han obtenido mediante el software estadístico SPSS.

${ }^{9}$ En ocasiones, también se ha postulado que el reajuste tonal puede darse no sólo con el último valor tonal del GE inmediatamente anterior, sino también con el primer valor tonal de este (Garrido, 2002). En cualquier caso, este no es el sentido en el que utilizamos reajuste en este estudio.
} 
antecedentes. Esto es, podemos encontrar GE que no tengan una pausa anterior, con lo que superamos la concepción de grupo fónico, es decir, "porción del discurso comprendida entre dos pausas" (Navarro Tomás, 1944: 41). De todos los grupos entonativos estudiados, la segmentación se realiza de la siguiente manera:

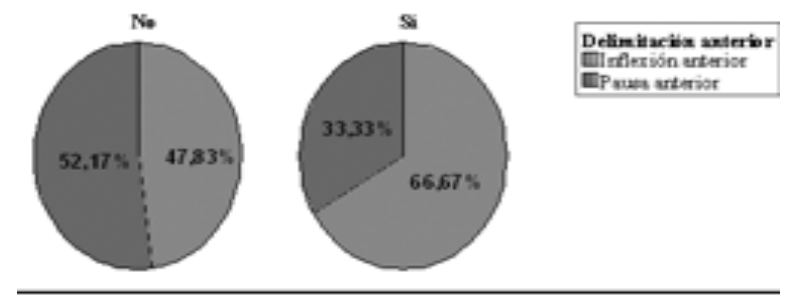

Figura 2. Grupos entonativos introducidos por inflexión tonal o pausa anterior

En la Fig.2, se advierte que hay un reparto equitativo en la manera de introducir los grupos entonativos en el habla habitual (52,17\% de pausas y 47,83\% de inflexiones), mientras que, en el caso de grupo con ED, parece más común su introducción mediante inflexiones tonales. En realidad, estos datos no aportan diferencias significativas entre unos grupos y otros y, por los porcentajes alcanzados, parece evidente que hay bastante equilibrio entre pausas e inflexiones a la hora de introducir grupos entonativos de cualquier tipo (con o sin ED). Así pues, la delimitación anterior no será una marca identificativa que distinga unos grupos de otros.

En otro orden de cosas, el hecho de que haya más ligazón entre GE con ED mediante inflexiones tonales podría hacernos pensar que hay más rapidez de habla. Si atendemos a la duración media de los grupos entonativos, encontramos los resultados de la siguiente tabla:

\begin{tabular}{|lc|c|c|}
\hline & \multicolumn{2}{|c|}{ Duración grupo entonativo } \\
& & Media & Mediana \\
\hline Estilo directo & No & 0,98 & 0,85 \\
& Sí & 1,11 & 1,08 \\
\hline
\end{tabular}

Figura 3. Duración media por grupo entonativo

Como ejemplifica la Fig.3, los grupos entonativos con ED son más amplios temporalmente que los GE habituales. Esto podría hacernos pensar que, al durar más, son más lentos desde un punto de vista elocutivo, sin embargo, hay que tener en cuenta que, en realidad, la distribución temporal tiene poco que aportar si no se tiene en cuenta el número 
de sílabas que se pronuncian en cada grupo entonativo. En nuestro corpus, la media de sílabas por GE con ED y sin ED es la siguiente:

\begin{tabular}{|ll|l|l|}
\hline \multirow{2}{*}{} & \multicolumn{2}{|l|}{ Sílabas por grupo entonativo } \\
\cline { 3 - 4 } & & Media & Mediana \\
\hline Estilo & No & 6,07 & 5,00 \\
directo & Sí & 6,93 & 7,00 \\
\hline
\end{tabular}

Figura 4. Media de sílabas por grupo entonativo

Por lo tanto, dado que conocemos la media de duración por grupo entonativo $(0,98$ segundos para grupos sin ED y 1,11 segundos para grupos con ED), podemos saber cuál es la velocidad de habla media por GE, dividiendo el número medio de sílabas por grupo entonativo por cada uno de los valores anteriores. Así, los GE sin ED tienen una velocidad media de 6,19 sílabas por segundo, mientras que, en GE con ED, son 6,24 sílabas por segundo.

Como puede apreciarse, no hay una diferencia sustancial en cuanto a velocidad de habla. Si bien, los GE con ED de nuestro corpus tienen una velocidad de habla superior, es apenas un $1 \%$ superior a los GE sin ED. En ese sentido, hasta que una investigación más detallada aporte nuevos datos, no podemos considerar que la delimitación anterior (inflexión o pausa) o la velocidad de habla sean criterios significativos para remarcar una diferencia entre secuencias con o sin estilo directo de habla.

Finalmente, es interesante observar en qué posición suelen aparecer los fragmentos con ED, ya que, de entrada, parece factible intuir que no aparezcan por norma en situación inicial de intervención. Así pues, en el corpus utilizado en este estudio hemos encontrado los siguientes datos:

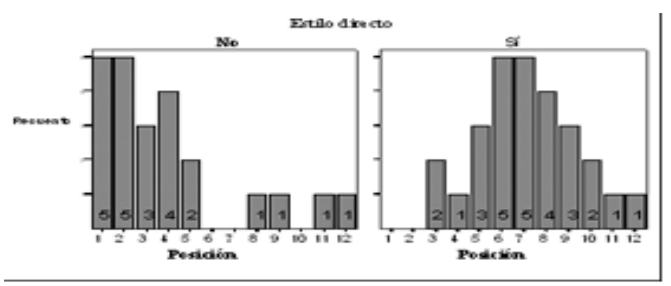

Figura 5. Distribución posicional de los $\mathrm{GE}^{10}$

${ }^{10}$ Los números que aparecen dentro de cada barra indican el número total (frecuencia absoluta) de grupos entonativos en cada posición, situada en el eje horizontal (1-12). 
Como se observa en la anterior figura, la intervención más extensa de nuestro estudio tiene 12 GE. Lo interesante del anterior gráfico es que los GE que forman parte de ED aparecen a partir de la $3^{\circ}$ posición y no antes. Además, es también común que en posición central (posiciones 6, 7 y 8) aparezcan GE con ED.

Así, este dato puede hacernos suponer que los fragmentos con ED suelen introducirse en el discurso cotidiano en situaciones posicionales intermedias ${ }^{11}$, bien para ejemplificar algo 0 mantener una secuencia narrativa (6), bien para aportar una dosis de intensificación dramática (7):

(6) lo tuvieronn no sé cuantas horas lo tuvimos sin tomar nada $\uparrow / /$ entonces luego nos dijo la enfermera $\uparrow$ a partir de la una de la mañana $\downarrow / /$ le vamos a dar agua... (Briz y Grupo Val.Es.Co., 2002: 195).

(7) pero luego lo he llevao a éste y dice $N O O /$ si fuese de ombligo $\uparrow /$ le dejaríamos que el niño... (Briz y Grupo Val.Es.Co., 2002: 192).

\subsection{Reajuste tonal}

Así las cosas, más allá de la F0 media y del rango tonal (criterios que sí nos sirven para caracterizar la singularidad del ED), existe un último fenómeno prosódico, el reajuste tonal, que puede ser útil a la hora de caracterizar el estilo habitual y el estilo directo de habla.

En primer lugar, debemos observar el número de reajustes que aparecen tanto en fragmentos que introducen ED, como en fragmentos normales de habla. Para poder observar dicho reparto con mayor claridad, observemos el siguiente gráfico:

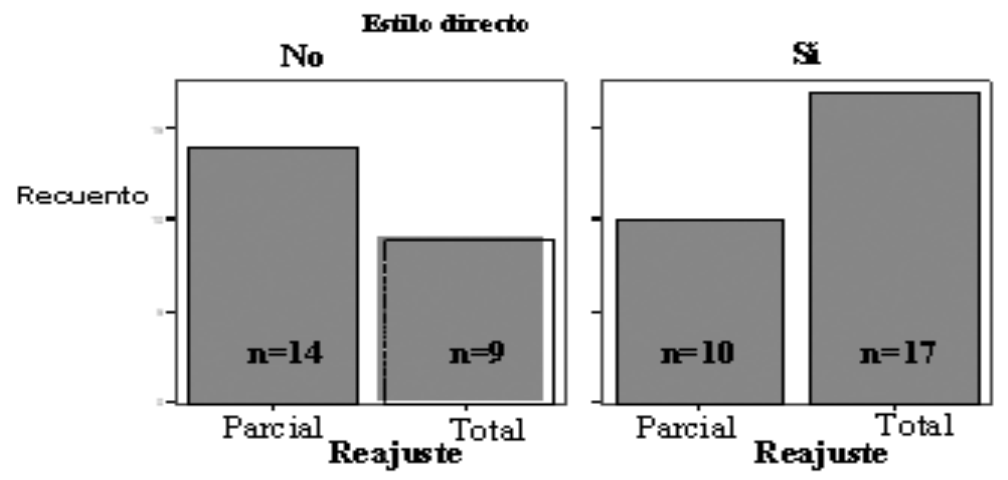

Figura 6. Reparto del tipo de reajuste en fragmentos con ED o sin ED

En la anterior figura, podemos observar que, inicialmente, para fragmentos con ED

\footnotetext{
${ }^{11}$ En nuestro corpus, no hemos recogido intervenciones que comiencen como en Vete de ahí tú me dijo el tonto ese, pero eso, evidentemente, no quiere decir que no existan, únicamente que, por la población analizada en este artículo, no parecen tan frecuentes como las posiciones intermedias.
} 
(señalados en el gráfico con un $\mathrm{Si}$ ) hay $17 \mathrm{GE}$ introducidos por reajuste total, y 9 con reajuste parcial, mientras que para fragmentos sin ED (señalados con $N o$ ), hay 10 con reajuste total y 14 con reajuste parcial. En total, así pues, en nuestro estudio tenemos 26 GE con ED y 24 sin ED.

En realidad, lo que nos interesa en esta investigación es determinar en cuántos casos, el reajuste total introduce GE iniciales de ED, es decir, grupos entonativos que suponen una transacción entre GE normales y GE de ED. Un ejemplo de ello puede observarse en la unión de los siguientes grupos entonativos:

(8) una compañera me dice ;hala!/ Carmen (Briz y Grupo Val.Es.Co., 2002: 191).

En el ejemplo anterior, el aspecto que queremos tratar en el reajuste tonal que opera en la parcela dice ;hala!, puesto que supone una frontera tonal entre un GE sin ED (...dice) y otro con ED (;hala!...).

En nuestro corpus, hay 6 casos en los que esto sucede, es decir, en las 5 intervenciones analizadas, hay 6 casos (prácticamente 1 caso por intervención) en los que existe un GE inicial de estilo directo. En unos casos, se trata de un acto completo y, en otros, de un subacto inicial. Para observarlo mejor, atendamos a la siguiente tabla:

\begin{tabular}{|c|c|c|c|c|}
\hline & \multicolumn{4}{|c|}{ Cambio de estilo normal a estilo directo } \\
\hline & \multicolumn{4}{|c|}{$1^{\circ} \mathrm{GE}$ de estilo directo al cambio } \\
\hline & \multicolumn{4}{|c|}{ Reajuste } \\
\hline & \multicolumn{2}{|r|}{ Parcial } & \multicolumn{2}{|r|}{ Total } \\
\hline & Núm & Subtabla N \% & Núm & Subtabla N \% \\
\hline $\begin{array}{cc}\text { Acto } & \text { Subacto inicial } \\
& \text { único }\end{array}$ & $\begin{array}{l}0 \\
1\end{array}$ & $\begin{array}{c}, 0 \% \\
16,7 \%\end{array}$ & $\begin{array}{l}2 \\
3\end{array}$ & $\begin{array}{l}33,3 \% \\
50,0 \%\end{array}$ \\
\hline
\end{tabular}

Figura 7. Reajuste tonal en la transacción a GE con estilo directo

Casualmente, el único reajuste parcial que introduce un acto único presenta una diferencia tonal con el GE inmediatamente anterior de $-1,92 \mathrm{~Hz}$, con lo cual, prácticamente podríamos decir que la $\mathrm{F} 0$ se mantiene y, por lo tanto, podría entenderse como un reajuste total subyacente, dado que el registro tonal que presenta es bastante elocuente al respecto.

En los otros 5 casos, por el contrario, hay una media tonal de $+66,08 \mathrm{~Hz}$ y de $+39,56 \mathrm{~Hz}$ de mediana, es decir, hay un incremento evidente entre el último GE sin estilo directo y el primero con estilo directo. De nuevo, se confirma lo que habíamos señalado con anterioridad, esto es, que los fragmentos con ED presentan niveles tonales mayores dentro del registro tonal de cada hablante.

Por el contrario, si observamos en conjunto el reparto de tipos de reajuste para fragmentos con ED y fragmentos sin ED, nos encontramos con algunos fenómenos llamativos. Para advertirlo con mayor claridad, introducimos la siguiente tabla: 


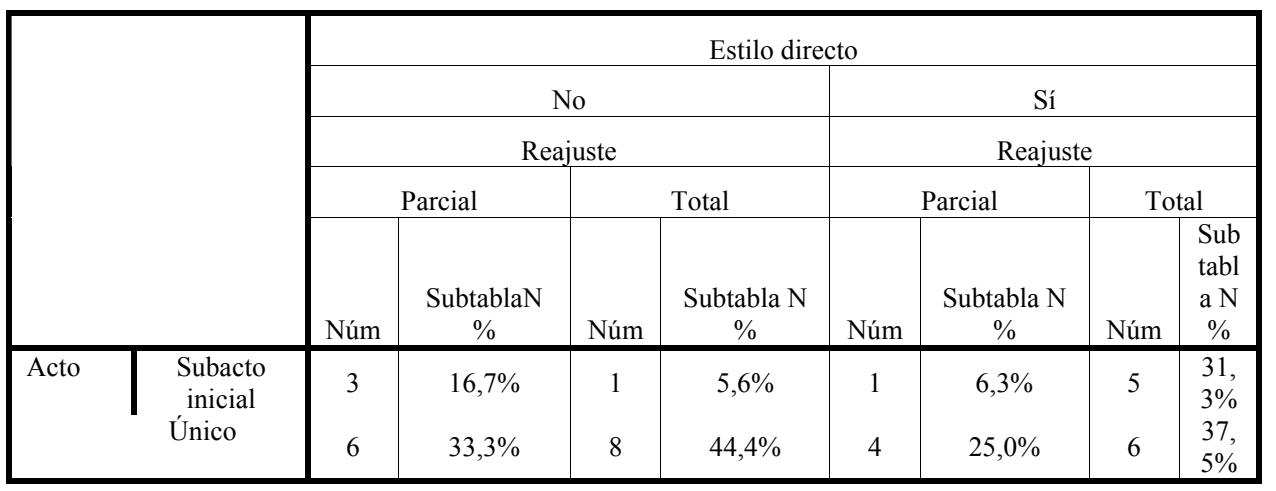

Figura 8. Reajuste total o parcial en subactos iniciales y actos únicos

En primer lugar, llama la atención el hecho de que el número de reajuste total y parcial en fragmentos sin ED sea equitativo (9 y 9), mientras que en fragmentos con ED, hay 5 GE introducidos por reajuste parcial que, a su vez, introducen un subacto inicial o un acto único y, por otro lado, $11 \mathrm{GE}$ con reajuste total. En esta tabla, así pues, hemos incluido todos los GE de los fragmentos con ED o sin ED, es decir, no nos hemos limitado, como en la tabla de la Fig.6, a aquellos GE que suponen un cambio de estilo normal a estilo directo de habla.

Evidentemente, no se puede concluir a partir de lo estudiado que, en fragmentos de habla habitual, no exista correlación entre reajuste total y comienzo de acto (sea único o subacto inicial) puesto que, para ello, se necesitaría una población más extensa; no obstante, lo que sí que es llamativo es que un $68,8 \%$ de los GE con ED estudiados son presentados tonalmente con un reajuste total y eso, a nuestro juicio, es debido a la mayor amplitud tonal de los fragmentos que presentan estilo directo.

\section{Conclusiones}

En general, a partir de lo postulado en este artículo, podemos concluir que hay ciertas peculiaridades prosódicas que configuran la articulación de fragmentos de habla que presentan estilo directo en su elocución. A partir de los objetivos previamente formulados y de la investigación realizada, podemos llegar a las siguientes conclusiones:

a) El estilo directo de habla no tiene una velocidad de habla claramente superior que la de otros fragmentos orales.

b) La delimitación anterior (mediante inflexión tonal o pausa) tampoco es un indicativo preciso para caracterizar la diferencia entre grupos entonativos con estilo directo y grupos habituales de habla.

c) Las secuencias con estilo directo no aparecen por norma en posición inicial de 
intervención y, además, suelen ubicarse en posiciones centrales para enfatizar el desarrollo narrativo de una anécdota o, en otro sentido, para simular el dictum y el modus de otros hablantes (presentes o no) y, con ello, acercar dramáticamente la acción al interlocutor.

d) La configuración tonal de los grupos fónicos sí es significativa en dos aspectos: en la F0 media y el rango tonal, ya que grupos entonativos con ED son superiores en gran medida a otros grupos entonativos sin ED. Esto se debe, como hemos señalado, a un mayor énfasis elocutivo y al hecho de que el hablante quiera distanciarse en cierta medida de lo dicho por otros; no tanto por el hecho de no estar de acuerdo con el dictum de esos otros (situación que podría darse), sino porque pretende, en la mayoría de lo casos, otorgar un valor de verosimilitud a sus palabras. Así pues, el uso de un tono mayor, además de enfático y demarcativo, pretende envolver el nivel segmental (las palabras), para conferir un matiz de credibilidad amparado en el $X$ habla asi o $Y$ lo diría así. Es evidente que, en muchas ocasiones, el nivel gestual también es muy importante para este fin.

e) El reajuste total es más frecuente para indicar la transacción entre grupos entonativos sin ED a grupos con ED. En cierto sentido, esta circunstancia se explica por la mayor amplitud tonal de los fragmentos orales con ED.

Por otro lado, los resultados de este estudio pueden confirmarse o desestimarse en investigaciones posteriores con muestras poblacionales mayores. No obstante, dado que el método de muestreo ha sido aleatorio ${ }^{12} \mathrm{y}$, por tanto, representativo de la variedad múltiple de la población, convenimos en señalar que un análisis de más casos se aproximará, en gran medida, a los resultados aquí presentados.

En conclusión, el hablante tiende a dramatizar sus enunciados ecoicos ${ }^{13}$ mediante el uso de diferentes fórmulas; algunas de ellas (reajuste total, amplitud tonal, F0 media superior, posición...) han sido tratadas en este artículo; asímismo, también existen otras que deben ser tenidas en cuenta para trabajos posteriores como, por ejemplo, la modulación de la voz o los gestos.

\footnotetext{
12 Las intervenciones y, posteriormente, los grupos entonativos analizados en este estudio han sido elegidos aleatoriamente puesto que se han seleccionado al azar de entre todas las intervenciones del Corpus de conversaciones coloquiales publicado en 2002 por Briz y Grupo Val.Es.Co. Por tanto, "cada elemento de la población tiene la misma probabilidad de salir elegido" (Peña y Romo, 1999: 269). Esto concede un alto grado de representatividad a los resultados de esta investigación.

${ }^{13}$ Los enunciados tipo eco se han abordado, entre otros, por la Teoría de la relevancia, es decir, enunciados de carácter metarepresentativo que intentan simular las palabras de uno mismo o de otras persona en una situación contextual pasada o imaginaria (Wilson 2000).
} 


\section{Referencias bibliográficas}

Antas, D. (2005): Auxiliar para el comentario de textos literarios. Barcelona, Octaedro.

Briz Gómez, A. y Grupo Val.Es.Co., (2002): Corpus de conversaciones coloquiales. Madrid, Arco Libros.

Garrido Almiñana, J.M. (2002): “La escuela holandesa: el modelo IPO”. En Prieto, P. (ed.), Teorías de la entonación. Barcelona, Ariel (97-121).

Hidalgo Navarro, A. (2006): "Estructura e interpretación en la conversación coloquial: el papel del componente prosódico", RFULL, 24, 129-151.

Hidalgo Navarro, A. y X. A. Padilla García (2006): "Bases para el análisis de las unidades menores del discurso oral: los subactos", Oralia, 9, 109-144.

Grupo Val.Es.Co (2003): "Un sistema de unidades para el estudio del lenguaje coloquial", Oralia, 6, 7-61.

Navarro Tomás, T. (1944): Manual de entonación española. Madrid, Guadarrama.

Peña, D. y J. Romo (1999): Introducción a la estadística para las Ciencias sociales. Madrid, McGraw-Hill.

Quilis, A. et alii (1993): "El grupo fónico y el grupo de entonación en español hablado", Revista de Filología Española, 73, 55-64.

Wennerstrom, A. (2001): Music of everyday speech: Prosody and discourse analysis. Cary, Oxford University Press.

Wilson, D. (2000): “Metarepresentation in linguistic communication”. En Sperber, D. (ed.), Metarepresentations. Oxford, Oxford University Press. 